\title{
SAFE Transport: Wearing Face Masks Significantly Reduces The Spread of COVID-19 On Trains
}

Hanna Grzybowska ( $\sim$ hanna.grzybowska@data61.csiro.au )

Data61

\section{Roslyn I. Hickson}

Health and Biosecurity, CSIRO

\section{Bishal Bhandari}

Data61

Chen Cai

Data61

Michael Towke

Transport for New South Wales

Benjamin Itzstein

Data61

Raja Jurdak

Queensland University of Technology

Kamran Najeebullah

Data61

\section{Adrian Plani}

Data61

\section{Ahmad El Shoghri}

Health and Biosecurity, CSIRO

Dean Paini

Health and Biosecurity, CSIRO

\section{Research Article}

Keywords: Covid19, SARS-CoV-2, Transit assignment, Disease spread model, Face masks.

Posted Date: February 16th, 2022

DOI: https://doi.org/10.21203/rs.3.rs-1290363/v1

License: (9) (1) This work is licensed under a Creative Commons Attribution 4.0 International License. 



\title{
SAFE Transport: Wearing Face Masks Significantly Reduces the Spread of COVID-19 on Trains
}

\author{
Hanna Grzybowska ${ }^{a, f}$, R.I. Hickson ${ }^{b, c}$, Bishal Bhandari $^{a}$, \\ Chen Cai ${ }^{a}$, Michael Towke ${ }^{d}$, Benjamin Itzstein ${ }^{a}$, Raja Jurdak ${ }^{e}$, Jessica Liebig ${ }^{b}$, \\ Kamran Najeebullah ${ }^{a}$, Adrian Plani ${ }^{a}$, Ahmad El Shoghri ${ }^{b}$, and Dean Paini ${ }^{b}$ \\ ${ }^{a}$ Data61, CSIRO, Australia \\ ${ }^{b}$ Health and Biosecurity, CSIRO, Australia \\ ${ }^{c}$ College of Public Health, Medical \& Veterinary Sciences, \\ and Australian Institute of Tropical Health and Medicine, \\ James Cook University, Townsville, Australia \\ $d$ Transport for New South Wales, Sydney, Australia \\ ${ }^{e}$ Queensland University of Technology, Brisbane, QLD, Australia \\ ${ }^{f}$ Research Centre for Integrated Transport Innovation, \\ School of Civil and Environmental Engineering, \\ University of New South Wales, Sydney NSW, Australia
}

\begin{abstract}
COVID-19 has had a substantial impact globally. It spreads readily, particularly in enclosed and crowded spaces, such as public transport carriages, yet there are limited studies on how this risk can be reduced. We developed a tool for exploring the potential impacts of mitigation strategies on public transport networks, called the Systems Analytics for Epidemiology in Transport (SAFE Transport). SAFE Transport combines an agent-based transit assignment model, a community-wide transmission model, and a transit disease spread model to support strategic and operational decision making. For this simulated COVID-19 case study, the transit disease spread model incorporates both direct (person-to-person) and fomite (person-to-surface-to-person) (personsurface-person) transmission modes. We determine the probable impact of wearing face masks on trains over a seven day simulation horizon, showing substantial and statistically significant reductions in new cases when passenger mask wearing proportions are greater than $80 \%$.
\end{abstract}

Keywords: Covid19; SARS-CoV-2; Transit assignment; Disease spread model; Face masks.

\section{Introduction}

In December 2019, a local outbreak of severe acute respiratory syndrome SARS-CoV-2, causing the disease referred to as COVID-19, in Wuhan (Hubei, China) became a pan- 
demic, spreading to 222 countries, with over 191 million confirmed cases and more than 4.1 million deaths to date [3]. The result has been a massive impact on the global economy including government and individual debt, slow down in national and international travel, tourism, commerce and trade, and sociological issues, amongst others (see, for example, $[27,28])$. It has effectively challenged the status quo of every day activities and ways of life. Another, more specific example is the change in the way people commute and use services such as public transport. Initially the substantial drop in public transport patronage was due to local or national lock-downs. However, with restrictions lifting in some countries, there has generally not been a concurrent increase in public transport use [4]. Globally, people are reluctant to use public transport for fear of contracting COVID-19. With the emergence of potentially more virulent COVID-19 variants that could reduce the effectiveness of vaccinations, it is of paramount importance that public transport operators are able to ensure their services are safe and then restore public confidence.

There are various non-pharmaceutical measures that can be applied to increase safety on public transport. These include, but are not limited to: introduction of more thorough and more frequent cleaning of the rolling stock, protecting the drivers and public transport operators with personal protective equipment (PPE), mandating wearing face masks by passengers, marking seats and standing spaces to maintain physical distances, forbidding front-door boarding to protect the drivers and encouraging boarding through other doors (particularly on buses [30]), lowering the maximum occupancy limits, encouraging hand sanitation, and shutting down stations shown to be hot-spots.

For researchers, the challenge is to show that these measures can be effective at reducing the spread of COVID-19 on public transport networks. Indeed there has been research that shows the survival time of the virus on different surfaces [8], the effectiveness of cleaning $[34,35]$, hand sanitation [32, 33], and lowering maximum occupancy limits [29, 30, 31]. Perhaps the most obvious measure would be the mandating of face masks for commuters, and while face masks have been generally accepted by most to be able to significantly reduce the spread of COVID-19 (see, for example, $[6,9]$ ), no work has attempted to quantitatively estimate the benefit of face masks on public transport. The benefit of such work could provide substantial reassurance to both government policy makers charged with ensuring the public's safety when they commute, and public commuters themselves. 
Here we present a tool, which can explore the probable impacts of such measures to better support strategic and operational decision making by public transport operators. The Systems Analytics for Epidemiology in Transport (SAFE Transport) tool combines an agent-based transit assignment model, a community-wide transmission model, and a transit disease spread model. The model has been tested on a set of artificial scenarios exploring the potential impact of non-pharmaceutical mitigation strategies such as face masks, using COVID-19 realistic values reported on other train networks. We focus on a case study of COVID-19 on the train network in Sydney, Australia, exploring the probable impacts of different proportions of passengers wearing face masks (that is, face mask coverage).

\section{Results}

To determine the probable impact of face mask wearing coverage by passengers, we focus on the total number of new COVID-19 infections over a 7 day time period as the output of interest. That is, the cumulative number of new infections over a 7 day simulation time horizon. We consider the following scenarios:

- Baseline - no mitigation strategies were applied (baseline)

- Face mask wearing by $25 \%$ of commuters (Mask_25)

- Face mask wearing by $50 \%$ of commuters (Mask_50)

- Face mask wearing by $75 \%$ of commuters (Mask_75)

- Face mask wearing by $80 \%$ of commuters (Mask_80)

- Face mask wearing by $100 \%$ of commuters (Mask_100)

To account for stochasticity in the underlying disease transmission process, each scenario was run 100 times, with summary statistics provided for the average and variation across all of these repeats. For each scenario, the relevant proportion of passengers were selected at random to wear a mask, independent of their disease progression status (that is, susceptible, exposed, infectious, or recovered). Further information is provided in $\S 3$ and the Supplementary materials ??. 


\section{$2.180 \%$ of passengers need to wear a mask to see a statistically significant reduction in 7 days}

The probable impacts of the different face mask wearing coverage levels over the full 7 day horizon are summarised by the Box Plot depicted in Figure 1. Note, for ease of visually identifying statistically significant differences, the whiskers display the $95 \%$ confidence intervals, as opposed to the traditional minimum and maximum. That is, if the top whisker of the mask wearing scenario is below the bottom whisker of the baseline, that level of mask wearing coverage is statistically significant over the course of the considered time horizon of 7 days. We demonstrate that the scenario with $80 \%$ of passengers wearing face masks shows a $68 \%$ reduction in total number of new infections and this is statistically significant when compared to the base case of no masks. In the case when $100 \%$ of passengers wear face masks, the reduction is $80 \%$ in comparison with the base case with no masks.

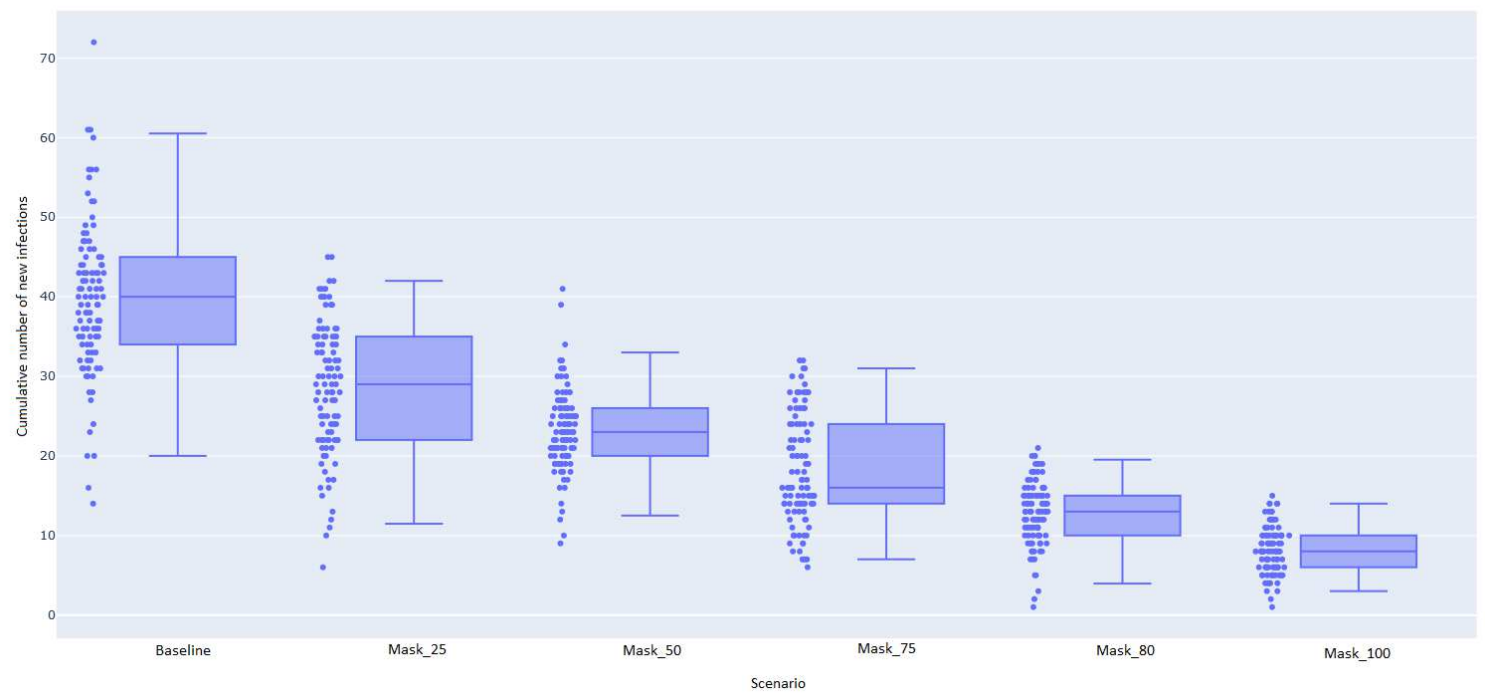

Figure 1: Total number of new infections after 7 days, for the different mask wearing coverage scenarios. Note: here the whiskers depict the $95 \%$ confidence intervals.

An important aspect of this modelling is that face masks do not perfectly prevent viral shedding (transmission from an infectious person) or prevent infection of those susceptible. This is reflected by there still being new cases, despite $100 \%$ mask wearing coverage. 


\subsection{Divergence between the baseline and mask wearing scenar- ios increases over time}

Figure 2 shows the total number of infections over time for all the mask wearing scenarios in comparison with the baseline. As the percentage of people wearing masks grows, the

$[\mathrm{a}]$
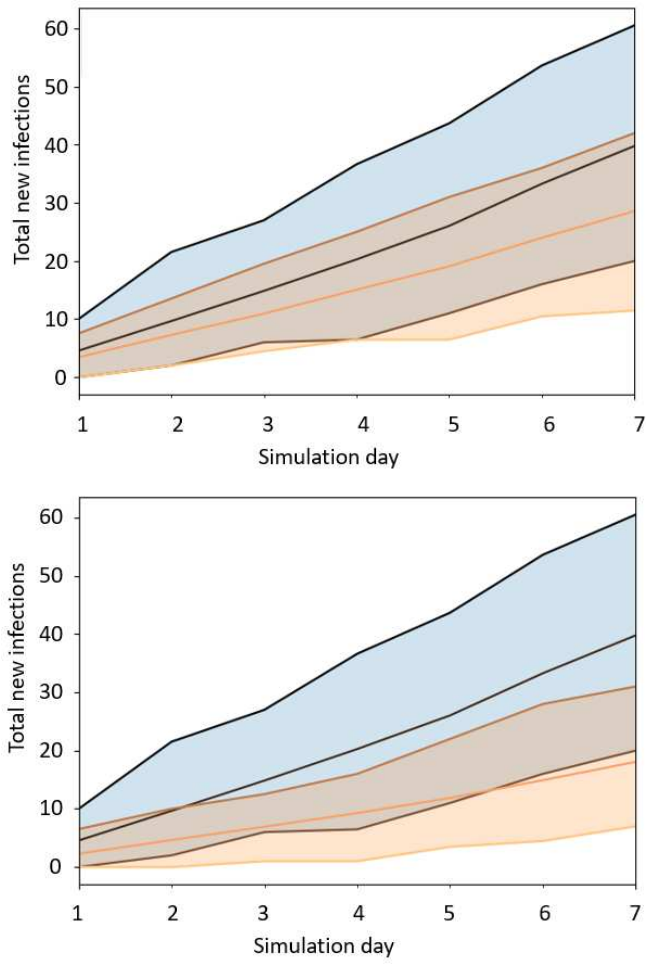

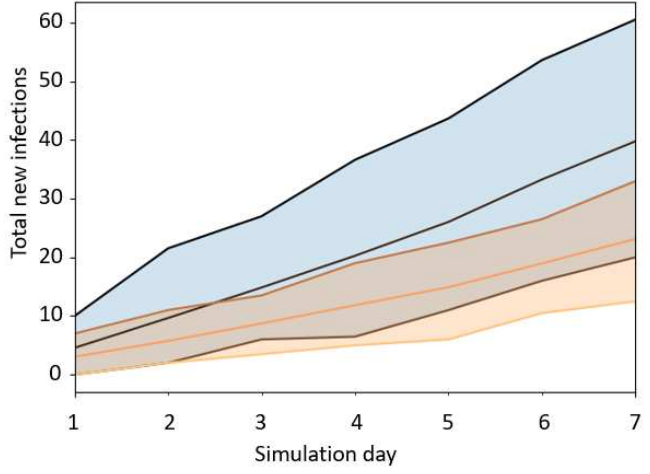

$[\mathrm{b}]$

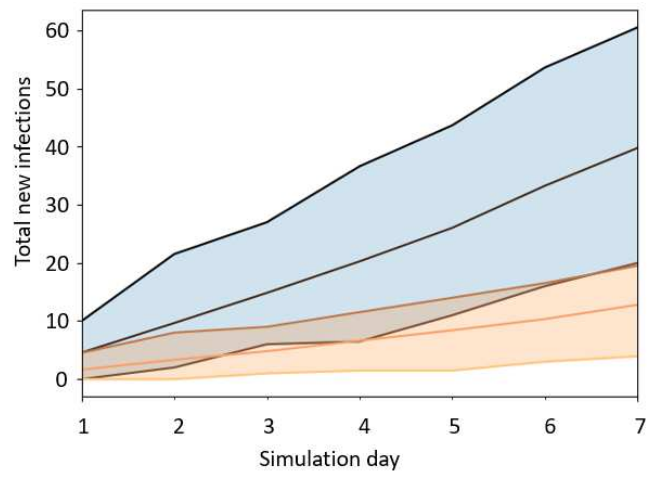

$[\mathrm{c}]$

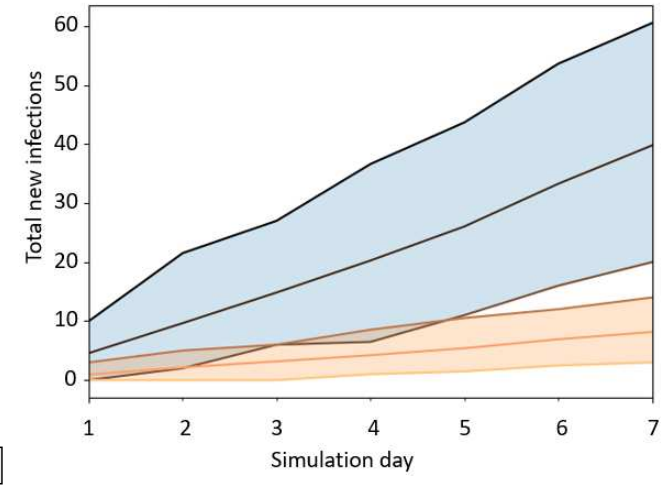

Figure 2: Comparison of the evolution in time of the total number of infections between the baseline (blue) and scenarios with face masks (orange) (average and 95\% confidence intervals): [a] baseline vs Mask_25, [b] baseline vs Mask_50, [c] baseline vs Mask_75, [d] baseline vs Mask_80, [e] baseline vs Mask_100. 
impact becomes more obvious over the 7 day time horizon. The higher the percentage of people wearing face masks, the sooner the results diverge significantly. For the $80 \%$ scenario, statistical significance does not occur until day 7 , while for the $100 \%$ scenario this occurs at day 5 . For a 7 day time frame then, the $80 \%$ scenario may be defined as a "tipping point". It is expected that for longer simulation horizons the two compared distributions would continue to diverge, and after sufficient time even the $25 \%$ scenario might become statistically significantly different to the baseline.

We have focused on a 7 day time horizon as a period when the general public would like to see positive improvement from expectations that they wear masks. However, this indicates that for more strategic planning, longer time horizons may be of interest.

\subsection{How robust are the mask wearing impacts to proportions of transmission via direct vs fomite routes?}

One of the key transmission model parameters is the proportion of virus that contributes to person-to-surface-to-person (fomite) transmission $\left(p_{s 0}\right)$, versus person-to-person (direct) transmission $\left(1-p_{s 0}\right)$. Figure 3 depicts how the average number of total infections after 7 days is affected by this parameter. The current evidence suggests aerosol transmission likely dominates (see, for example, $[36,37,13,38]$ ), and so the proportion of virus shed by infectious passengers that contributes to fomite transmission when no mask is worn is expected to result in small values of $p_{s 0}$. We find that $100 \%$ mask coverage results in a statistically significant reduction of new cases after 7 days when $p_{s 0} \lesssim 0.125$ (Figure $3 \mathrm{~b}$ ). Further, we find $80 \%$ mask coverage only results in a statistically significant reduction in the average number of new cases after 7 days for values of $p_{s 0} \leq 0.1$ (Figure 3a).

\section{Methods}

We developed a modelling framework to capture the key components of pathogen transmission on public transport networks. This is a general framework that could be used for a wide variety of pathogens, transport modes (including multi-modal) and public trans-

port networks, though here we focus on COVID-19 transmission on trains. An overview of the modelling framework is provided in Figure 4. There are four main components: 

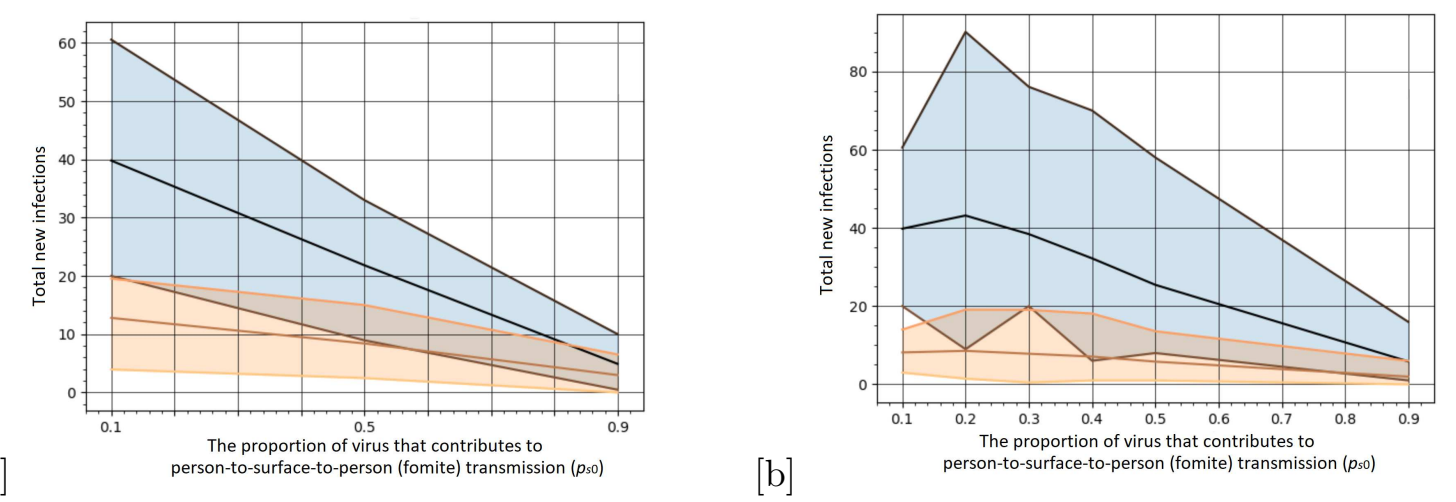

Figure 3: Comparison of how the total number of infections is affected by the proportion of virus contributing to fomite transmission in the absence of a mask $\left(p_{s 0}\right)$ (in blue). The lines depict the average, and the shaded region indicate the $95 \%$ confidence intervals based on 100+ simulations; (a) $80 \%$ mask coverage, (b) 100\% mask coverage (in orange) (Note: for $p_{s 0}=0.2$ and 0.3 there are 200 repeat simulations to capture the stochastic variation).

1. Transit Assignment Engine: a transit assignment engine simulating the movement of passengers in the public transport network, for our case study this is the train network in Sydney, Australia;

2. Modelling the Spread of Infection: the transit disease spread model, for our COVID19 case study this incorporates two forms of transmission: direct (person-to-person) and fomite (person-to-surface-to-person);

3. Outputs: an analytical module providing summary statistics and visualisations of results; and

4. Disease Seeding: a community-wide transmission model informing the disease seeding (that is, the expected number of infectious passengers travelling on the Sydney train network).

In addition to these components, our modelling framework requires input data and specification of the scenarios of interest (such as including the non-pharmaceutical interventions being considered).

In our case study, this framework involves assigning a passenger to a train trip leg, based on travel smart card data and an assigned shortest feasible path. Each leg of the trip is 


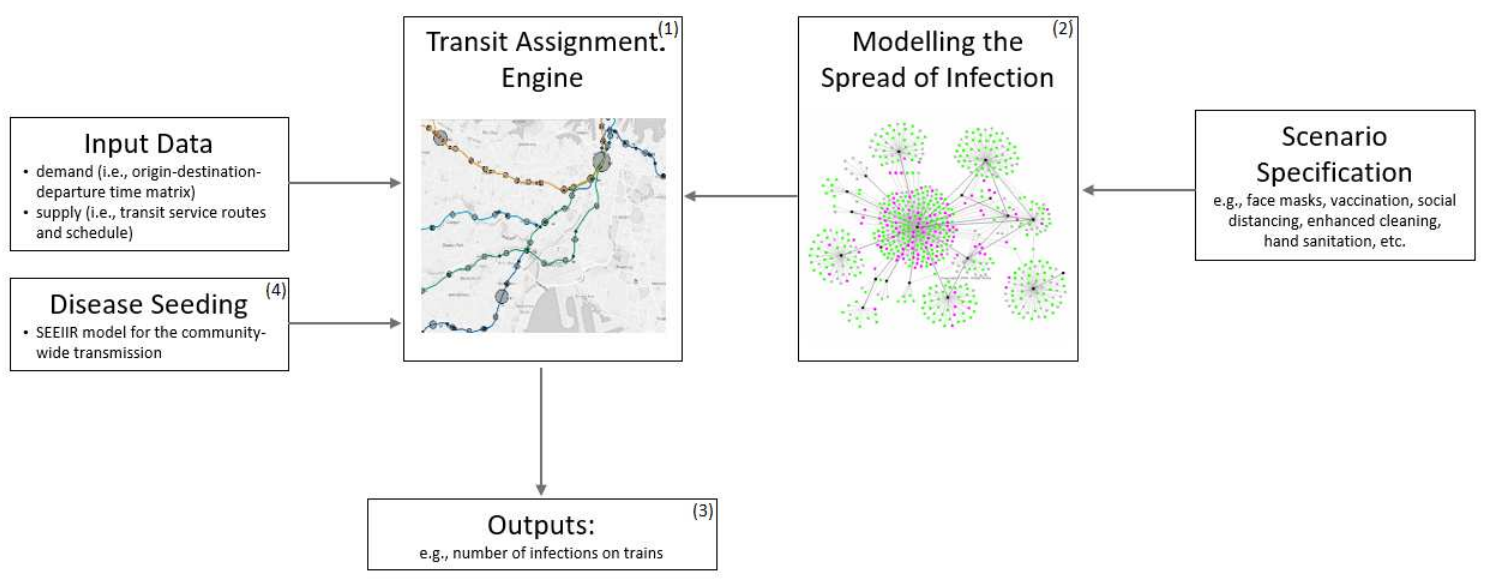

Figure 4: Modelling framework, with more information on each component outlined in $\S 3.1-3.3$. The modular structure allows for flexibility in designation of geographical location, pathogen of interest, and scenarios explored. The agent-based model yields detailed outputs to inform operational and strategic decisions.

assigned to a transit service vehicle (in this case, a train), according to a specified capacity and current occupancy. The trains operate in accordance with a pre-defined schedule. Every time a passenger finishes their trip, the transit disease model is triggered and this passenger is assigned an exposure status (that is, whether the passenger was infected during their trip). The information about new infections is sent to the visualisation module to be displayed on a dashboard. At the end of every simulated day and at the end of the simulation horizon, the total number of new infections is calculated and reported for final evaluation. The SAFE Transport modules are further described in subsections 3.1-3.3.

\subsection{Transit Assignment Engine}

The transit assignment engine is an agent-based simulation platform, which maps trip demand to transit service supply. The transit assignment engine underpins SAFE Transport, as it provides the network of contacts between travelling passengers (that is, with whom and for how long they are in contact).

The engine depends on input data in the form of:

1. trip demand (that is, trip origin, trip destination and start trip time for every passenger), and 
2. transit service supply (that is, routes and schedules of public transport services).

The full architecture is provided in the Supplement ??. The total number of trips in Sydney's train network before COVID-19 was about 8 million per week. To account for the patronage drop during COVID-19, we assume the demand to be $10 \%$ of the total which aligns with the real-life observations $[20,19,21]$. The supply of services did not change in Sydney due to COVID-19. Trip demand is captured by smart cards, including tap-on and tap-off data with location GPS coordinates and timestamp. This is used by the shortest path router to calculate a set of time-dependent shortest paths for each travelling passenger using the classic Dijkstra algorithm. Throughout the simulation the passengers are tracked and detailed dynamic outputs are collected for every: agent, link (between two consecutive stops/stations), stop/station, service vehicle, service line, and the whole network.

\subsection{Modelling the Spread of Infection on Trains}

At its core, the disease spread model on trains provides a probability of becoming infected for each susceptible passenger, based on the current and past travel of infectious passengers in the same spatial area. The model uses a number of simplifying assumptions, the most important being that we ignore any age-based effects (all agents are identical), and we assume homogeneity of mixing within the spatial area considered, including equal distribution of passengers throughout the train. In part to account for our homogeneous mixing within a spatial area assumption, we use a half-carriage spatial area for our model, due to the stack structure (including upper and lower deck) of the Sydney train carriages (Waratah design).

The overall probability of a susceptible individual being infected is based on the standard probabilistic statement of being one minus the probability that they were not infected. This allows us to consider the probability of infection from each of the infectious passengers, and the surfaces on the half-carriage, separately. The transmission model uses the regression expression for the attack rate from the study by $\mathrm{Hu}$ et al. [7] (Fig 4 in [7], Average of all seats), both directly for the empirical person-to-person part of our transmission model, and for calibration of the mechanistic person-to-surface-to-person model. The mechanistic model is based on the transmission route model developed by Atkinson and Wein [5]. This approach models proportion of the virus shed drops to the surface, and the effective 
dose based on surface concentration. We then use a standard dose-response model for the probability of infection from contaminated surfaces (fomites). Further details of the transmission model are provided in the Supplement ??, along with sensitivity analyses of key parameters.

\subsection{Seeding}

Our primary disease spread model, as outlined in $\S 3.2$, only considers transmission on the public transport network (and is specifically calibrated to trains). To keep the numbers of infectious passengers travelling on trains identical across simulations for comparability, we use a deterministic compartmental model to approximate the transmission dynamics in the general community.

We use the standard susceptible-exposed-infectious-recovered progression structure, with the exposed and infectious compartments repeated to better account for the distribution of time spent in those states (see, for example, [26]). We refer to this as an "SEEIIR" model for short. We start the deterministic SEEIIR model with 2000 infectious cases, use the population of Sydney of 5.73 million in 2019 [18], and a basic reproduction number of $2.5[22,23]$. We used estimates of the proportion of the population who commute via public transport (approximately 20\% according to Census 2006, 2001 and 2016 data $[14,16]$ ), and the proportion of commuters who use trains (approximately $50.9 \%$ [15]), to arrive at an estimate of $10 \%$ of the population using trains in Sydney. Further details are provided in the Supplement ??, including a table with the numbers used to seed infectious passengers for each of the 7 days.

\subsection{Face Mask Wearing Scenarios}

The primary objective of this work was to explore the probable impacts of different face mask wearing proportions by passengers (that is, face mask coverage). How the mask wearing status of passengers effects the transit transmission model depends on whether the passenger is susceptible or infectious. The mask wearing status of infectious individuals reduces viral shedding. The mask wearing status of susceptible individuals is known to reduce the overall probability of infection [9], but does this through two separate mechanisms: (i) it explicitly effects the probability of being infected from the direct transmission 
component, and (ii) it effects the effective dose from the surface for the fomite transmission component.

To parameterise the viral shedding effect on those infectious passengers, we use the filtration efficacy of a two-layer cloth mask, as described in Howard et al. [6]. They found two-layer cloth masks provide an 88-94\% reduction in infectious particle load, and an 80$90 \%$ filtration efficacy. In our modelling we use $90 \%$ as it is within both of these bounds, and reduction in infection particle load is arguably the most important aspect.

For the reduction in susceptibility, the model was calibrated using a minimisation of the sum of squared errors to achieve the reported odds ratio of 0.22 for wearing a mask [9]. There is further information on this provided in the Supplement ??.

\section{Discussion}

The SAFE Transport tool presented here can support public transport agencies in both strategic and operational decision-making regarding disease spread mitigation options. Here we have applied it to train networks for informing COVID-19 related mitigation options. Hence the SAFE Transport tool is capable of contributing to recovery and resilience postpandemic.

We have shown that for this parameterisation of a COVID-19 disease on trains, if more than $80 \%$ of passengers wear face masks there is a substantial (68\% relative) and statistically significant reduction in the total number of new cases over a 7 day period. While this result is highly sensitive to the proportion of virus shed contributing to fomite vs direct transmission, recent evidence $[36,37]$ suggests fomite transmission is only a small contributor, and that indeed the proportion is speculatively around $10 \%$ (equivalent to the value of $p_{s 0}=0.1$, used here), and likely to be even smaller [38]. This finding and subsequent sensitivity analysis demonstrates the utility of our SAFE Transport tool, for a nuanced way of informing decision making with respect to disease spread on public transport networks.

We made a number of simplifying assumptions with respect to the disease transmission models. However, we explored the effect of most of these assumptions. The effect of the key parameter of the model on our outcome of interest, total number of new cases, is shown in $\S 2.3$, and the rest are shown in the Supplement ??. We assumed masks worn had the efficacy 
of a two-layer cloth mask. However, in the case when mask wearing has been mandated, even if some people are wearing less effective masks we would still expect to see a substantial and statistically significant reduction in new cases since our threshold for significance is 80\%. Our transmission model does not explicitly explore aerosol transmission, but this is implicitly included in the direct and fomite transmission being calibrated to recover the attack rate on trains reported by $\mathrm{Hu}$ et al. [7]. One major assumption made and not tested was the lack of age structure, which we ignored since our focus here has been on the use of adult commuters as primary train users [24].

Although our study was calibrated to trains (specifically to a half-carriage), policymakers and public transport operators could mandate the use of face masks on other forms of public transport with confidence that this will help reduce spread. A similar effect is expected for other public transport modes such as buses and trams, given their relatively similar physical attributes. For example, the sitting areas in public transport modes are organised in a similar pattern and proximity within a closed space.

If the travel demand for public transport returns to the pre-COVID-19 levels, maintaining the recommended distance of 1.5 metres between passengers [25] would be a challenge on any public transport mode. However, we would expect that while face masks would be mandatory in that situation, it would not be the only public health measure used to reduce the spread of COVID-19, as even with $100 \%$ of passengers wearing masks, we still show a low spread of COVID-19 . We therefore strongly support the "Swiss cheese" approach to mitigation and control efforts (see, for example, [39]).

There are still many unanswered questions and avenues of research with regards to reducing the spread of COVID-19 on public transport networks, and in restoring public confidence. Our SAFE Transport tool has significant potential, both in helping to answer specific COVID-19 questions, and also for other infectious diseases. In particular, the SAFE Transport tool is designed for a nuanced approach to informing operational and strategic decision making for public transport networks. Other areas of consideration that could be investigated with the SAFE Transport tool include: (i) understanding how cleaning regimes affect the transmission dynamics (and how sensitive this is to proportion of transmission that is direct vs fomite), (ii) understanding how other COVID-19 variants, such as Delta might be affected by non-pharmaceutical strategies, (iii) determining general transmission 
patterns and in particular hot-spots on the network and how they are affected by different measures, (iv) explicit modelling of other public transport types to confirm the applicability of our findings across general networks, (v) better understand spatial distribution of passengers within the public transport vehicles (such as carriages for trains) and effects of different passenger densities, (vi) exploring longer simulation time horizons, and perhaps most importantly, (vii) including community vaccination levels to determine the need or effectiveness of non-pharmaceutical strategies.

In its design, the proposed modelling framework for SAFE Transport is generic and agnostic to transport networks and travel modes. It effectively enables the development of a digital twin of any public transport network (and any transport mode), providing a holistic view of its performance (for example, identify hot-spots) and the dynamics of a contagious disease spread.

\section{Conclusion}

In conclusion, we have developed a flexible framework for supporting operational and strategic decision making for disease transmission mitigation measures on public transit networks. The framework allows for building a digital twin of any transport network of any city, represent various modes of transport (including multi-modal), modelling various transmissible diseases and exploratory scenarios. This provides an opportunity for multitude of further investigative studies.

Our case study of mask wearing scenarios shows substantial and statistically significant impact on COVID-19 transmission, for at least 80\% coverage levels in a 7 day time period. We also found that higher levels of mask coverage result in earlier reduction in disease spread risk.

\section{Declarations}

\subsection{Ethics approval and consent to participate}

The paper has been submitted with full responsibility, following due ethical procedure, and there is no duplicate publication, fraud, plagiarism, or concerns about animal or human 
experimentation.

\subsection{Consent for publication}

Not applicable.

\subsection{Availability of data and material}

For data and material-related inquiries, please contact the corresponding author: Hanna Grzybowska.

Contact: hanna.grzybowska@data61.csiro.au

Affiliation: Data61, CSIRO, Australia, and

School of Civil and Environmental Engineering, University of New South Wales, Sydney NSW, Australia

\subsection{Competing interests}

No, I declare that the authors have no competing interests as defined by BMC, or other interests that might be perceived to influence the results and/or discussion reported in this paper.

\subsection{Funding}

This project received funding from Transport for New South Wales, Australia.

\subsection{Authors' contributions}

Hanna Grzybowska (corresponding author)

- transport modelling

- paper writing

- scenario design

- simulation design, development and runs 
- input data and results analysis

\section{R.I. Hickson}

- epidemiological modelling

- paper writing

- scenario design

- results interpretation

- code review

\section{Bishal Bhandari}

- implementation and deployment of the models in software environment

- code reviews

\section{Chen Cai}

- contribution to modelling and scenarios definition

- paper review

- results interpretation

\section{Michael Towke}

- contribution to modelling and scenarios definition

- paper review

\section{Benjamin Itzstein}

- implementation and deployment of the models in software environment

- code reviews

\section{Raja Jurdak}

- contribution to modelling and scenarios definition 
- paper review

Jessica Liebig

- contribution to modelling and scenarios definition, including model parameterisation

\section{Kamran Najeebulla}

- contribution to modelling and scenarios definition, including model parameterisation

\section{Adrian Plani}

- implementation and deployment of the models in software environment

\section{Ahmad El Shoghri}

- contribution to modelling and scenarios definition

\section{Dean Paini}

- contribution to modelling and scenarios definition

- paper writing

- results interpretation

\subsection{Acknowledgements}

The authors acknowledge the generous support from Transport for New South Wales, Australia.

\subsection{Authors Information (optional)}

Hanna Grzybowska (corresponding author)

Contact: hanna.grzybowska@data61.csiro.au

Affiliation: Data61, CSIRO, Australia, and

School of Civil and Environmental Engineering, University of New South Wales, Sydney NSW, Australia 


\section{R.I. Hickson}

Contact: roslyn.hickson@csiro.au

Affiliation: Health and Biosecurity, CSIRO, Australia, and

College of Public Health, Medical \& Veterinary Sciences, and

Australian Institute of Tropical Health and Medicine, James Cook University, Townsville, Australia

\section{Bishal Bhandari}

Contact: bishal.bhandari@data61.csiro.au

Affiliation: Data61, CSIRO, Australia

\section{Chen Cai}

Contact: chen.cai@data61.csiro.au

Affiliation: Data61, CSIRO, Australia

\section{Michael Towke}

Contact: michael.towke@transport.nsw.gov.au

Affiliation: Transport for New South Wales, Sydney, Australia

\section{Benjamin Itzstein}

Contact: ben.itzstein@data61.csiro.au

Affiliation: Data61, CSIRO, Australia

\section{Raja Jurdak}

Contact: r.jurdak@qut.edu.au

Affiliation: Queensland University of Technology, Brisbane, QLD, Australia

\section{Jessica Liebig}

Contact: jess.liebig@csiro.au

Affiliation: Health and Biosecurity, CSIRO, Australia 


\section{Kamran Najeebulla}

Contact: kamran.najeebullah@data61.csiro.au

Affiliation: Data61, CSIRO, Australia

\section{Adrian Plani}

Contact: adrian.plani@outlook.com

Affiliation: Data61, CSIRO, Australia

\section{Ahmad El Shoghri}

Contact: ahmad.elshoghri@data61.csiro.au

Affiliation: Health and Biosecurity, CSIRO, Australia

\section{Dean Paini}

Contact: dean.paini@csiro.au

Affiliation: Health and Biosecurity, CSIRO, Australia

\section{References}

[1] World Health Organization. Statement regarding cluster of pneumonia cases in Wuhan, China. Jan 9, 2020. https://www.who.int/china/news/detail/09-01-2020-whostatement-regardingcluster-of-pneumonia-cases-in-wuhan-china (last accessed: Feb 11, 2020).

[2] World Health Organization. Coronavirus disease 2019 (COVID-19) situation reports. https://www.who.int/ emergencies/diseases/novelcoronavirus-2019/situationreports (last accessed: Feb 17, 2020).

[3] World Health Organization. WHO COVID-19 Dashboard. Geneva: World Health Organization, 2020. https://covid19.who.int/ (last accessed: 22 Jul 2021)

[4] Google. Covid-19 Community Mobility Trends - Last updated 1 February 2021. https://ourworldindata.org/covid-mobility-trends (last accessed: 28 Jul 2021) 
[5] Atkinson, M.P. and Wein, L.M. 2008. Quantifying the routes of transmission for pandemic influenza. Bulletin of mathematical biology, 70(3), pp.820-867.

[6] Howard, J., Huang, A., Li, Z., Tufekci, Z., Zdimal, V., van der Westhuizen, H.M., von Delft, A., Price, A., Fridman, L., Tang, L.H., Tang, V., Watson, G.L., Bax, C.E., Shaikh, R., Questier, F., Hernandez, D., Chu, L.F., Ramirez, C.M., and Rimoin, A.w. 2021. An evidence review of face masks against COVID-19. Proceedings of the National Academy of Sciences of the United States of America. Vol. 118 No. 4 e2014564118. https://doi.org/10.1073/pnas.2014564118

[7] Hu, M., Lin, H., Wang, J., Xu, C., Tatem, A.J., Meng, B., Zhang, X., Liu, Y., Wang, P., Wu, G. and Xie, H. 2020. The risk of COVID-19 transmission in train passengers: an epidemiological and modelling study. Clinical Infectious Diseases.

[8] Riddell, S., Goldie, S., Hill, A., Eagles, D. and Drew, T.W. 2020. The effect of temperature on persistence of SARS-CoV-2 on common surfaces. Virology Journal,17(1), pp.1-7.

[9] Wang, Y., Tian, H., Zhang, L., Zhang, M., Guo, D., Wu, W., Zhang, X., Kan, G.L., Jia, L., Huo, D. and Liu, B. 2020. Reduction of secondary transmission of SARS-CoV-2 in households by face mask use, disinfection and social distancing: a cohort study in Beijing, China. BMJ Global Health, 5(5), p.e002794.

[10] Zhang, X. and Wang, J. 2020. Deducing the Dose-response Relation for Coronaviruses from COVID-19, SARS and MERS Meta-analysis Results. MedRxiv.

[11] Tang, B., Bragazzi, N.L., Li, Q., Tang, S., Xiao, Y. and Wu, J. 2020. An updated estimation of the risk of transmission of the novel coronavirus (2019-nCov). Infectious disease modelling, 5, pp.248-255.

[12] Chu, D.K., Akl, E.A., Duda, S., Solo, K., Yaacoub, S., Schünemann, H.J., El-harakeh, A., Bognanni, A., Lotfi, T., Loeb, M. and Hajizadeh, A. 2020. Physical distancing, face masks, and eye protection to prevent person-to-person transmission of SARS-CoV-2 and COVID-19: a systematic review and meta-analysis. The Lancet. 
[13] Meyerowitz, E.A., Richterman, A., Gandhi, R.T. and Sax, P.E. 2020. Transmission of SARS-CoV-2: a review of viral, host, and environmental factors. Annals of internal medicine.

[14] O'Sullivan, M. 2017. Sydneysiders the nation's biggest users of public transport for work commute. The Sydney Morning Herald. https://www.smh.com.au/national/nsw/sydneysiders-thenations-biggest-users-of-public-transport-for-work-commute-20171023gz632p.html\#: :text=Sydney\%20tops\%20the\%20nation\%20for\%20the\%20proportion\%20of ,buses\%2C\%20trams\%20or\%20ferries\%20for\%20their\%20daily\%20commute (last accessed: 25 Jun 2021)

[15] Wikipedia. 2020. Transport for NSW patronage in Sydney by mode. https://en.wikipedia.org/wiki/Template:Transport_for_NSW_patronage_in_Sydney_by_mode (last accessed: 25 Jun 2021)

[16] Australian Bureau of Statistics. 2008. Public transport use for work and study. https://www.ausstats.abs.gov.au/ausstats/subscriber.nsf/0/4028BBADB558AFFCCA 25748E0012AEEF/\$File/41020_2008_23.pdf (last accessed: 28 Jul 2021)

[17] Australian Bureau of Statistics. 2016. Australian Social Trends. https://www.abs.gov.au (last accessed: 28 Jul 2021)

[18] Australian Bureau of Statistics. 2016. 2016 Census QuickStats. https://quickstats.censusdata.abs.gov.au/census_services/getproduct/census/2016/ quickstat/1030 (last accessed: 28 Jul 2021)

[19] Sydney Morning Herald. 2021. Sydney's public transport patronage at lowest levels since 1800s. https://www.smh.com.au/national/nsw/sydney-s-public-transportpatronage-at-lowest-levels-since-1800s-20210719-p58b2v.html (last accessed: 19 Jul 2021)

[20] Sydney Morning Herald. 2020. The 51 million times Sydneysiders didn't tap on in March. https://www.smh.com.au/national/nsw/the-51-million-times-sydneysidersdidn-t-tap-on-in-march-20200408-p54i4z.html (last accessed: 27 Jul 2021) 
[21] Opendata Transport for New South Wales. 2021. Opal Patronage. https://opendata.transport.nsw.gov.au/dataset/opal-patronage (last accessed: 27 Jul 2021)

[22] Billah, A., Miah, M. and Khan, N. 2020. Reproductive number of coronavirus: A systematic review and meta-analysis based on global level evidence. PLOS ONE. https://doi.org/10.1371/journal.pone.0242128

[23] Liu, Y., Gayle, A.A., Wilder-Smith, A. and Rocklöv, J. 2020. The reproductive number of COVID-19 is higher compared to SARS coronavirus. Journal of Travel Medicine, Volume 27, Issue 2. https://doi.org/10.1093/jtm/taaa021 (last accessed: 27 Jul 2021)

[24] Transport for New South Wales. 2021. Train Patronage - Card Type. https://www.transport.nsw.gov.au/data-and-research/passenger-travel/trainpatronage/train-patronage-card-type (last accessed: 27 Jul 2021) (last accessed: 26 Jul 2021)

[25] Australian Government. Department of Health. 2021. Physical distancing for coronavirus (COVID-19). https://www.health.gov.au/news/health-alerts/novel-coronavirus2019-ncov-health-alert/how-to-protect-yourself-and-others-from-coronavirus-covid19/physical-distancing-for-coronavirus-covid-19 (last accesssed: 28 Jul 2021)

[26] Matt J. Keeling and Pejman Rohani. 2008. Modeling Infectious Diseases in Humans and Animals. Princeton University Press, 2008.

[27] Chowdhury, Emon Kalyan, Iffat Ishrat Khan, and Bablu Kumar Dhar. "Catastrophic Impact of Covid-19 on the Global Stock Markets and Economic Activities." Business and Society Review n/a, no. n/a. Accessed August 3, 2021. https://doi.org/10.1111/basr.12219.

[28] Verschuur, Jasper, Elco E. Koks, and Jim W. Hall. "Observed Impacts of the COVID19 Pandemic on Global Trade." Nature Human Behaviour 5, no. 3 (March 2021): 305-7. https://doi.org/10.1038/s41562-021-01060-5. 
[29] Sun, Chanjuan, and Zhiqiang Zhai. "The Efficacy of Social Distance and Ventilation Effectiveness in Preventing COVID-19 Transmission." Sustainable Cities and Society 62 (November 1, 2020): 102390. https://doi.org/10.1016/j.scs.2020.102390.

[30] Kamga, Camille, and Penny Eickemeyer. "Slowing the Spread of COVID19: Review of 'Social Distancing' Interventions Deployed by Public Transit in the United States and Canada." Transport Policy 106 (June 1, 2021): 25-36. https://doi.org/10.1016/j.tranpol.2021.03.014.

[31] Coppola, Pierluigi, and Francesco De Fabiis. "Impacts of Interpersonal Distancing OnBoard Trains during the COVID-19 Emergency." European Transport Research Review 13, no. 1 (February 9, 2021): 13. https://doi.org/10.1186/s12544-021-00474-6.

[32] Singh, Pallavi, Ipshita Potlia, Shitanshu Malhotra, Himangi Dubey, and Himanshu Chauhan. "Hand Sanitizer an Alternative to Hand Washing-A Review of Literature." Journal of Advanced Oral Research 11, no. 2 (November 1, 2020): 137-42. https://doi.org/10.1177/2320206820939403.

[33] Golin, Andrew P., Dexter Choi, and Aziz Ghahary. "Hand Sanitizers: A Review of Ingredients, Mechanisms of Action, Modes of Delivery, and Efficacy against Coronaviruses." American Journal of Infection Control 48, no. 9 (September 1, 2020): 1062-67. https://doi.org/10.1016/j.ajic.2020.06.182.

[34] CDC. "Community, Work, and School." Centers for Disease Control and Prevention, February 11, 2020. Last accessed 03 August 2021. https://www.cdc.gov/coronavirus/2019-ncov/community/disinfecting-buildingfacility.html.

[35] Elbadawy, Hossein M., Amin Khattab, Ali Alalawi, Fahad Dakilallah Aljohani, Hamza Sundogji, Ameira S. Mahmoud, Meky Abouzied, et al. "The Detection of SARS-CoV-2 in Outpatient Clinics and Public Facilities during the COVID-19 Pandemic." Journal of Medical Virology 93, no. 5 (2021): 2955-61. https://doi.org/10.1002/jmv.26819.

[36] Tang, J. W., W. P. Bahnfleth, P. M. Bluyssen, G. Buonanno, J. L. Jimenez, J. Kurnitski, Y. Li, et al. "Dismantling Myths on the Airborne Transmission of Severe Acute 
Respiratory Syndrome Coronavirus-2 (SARS-CoV-2)." Journal of Hospital Infection 110 (April 1, 2021): 89-96. https://doi.org/10.1016/j.jhin.2020.12.022.

[37] Greenhalgh, Trisha, Jose L Jimenez, Kimberly A Prather, Zeynep Tufekci, David Fisman, and Robert Schooley. "Ten Scientific Reasons in Support of Airborne Transmission of SARS-CoV-2." The Lancet 397, no. 10285 (May 1, 2021): 1603-5. https://doi.org/10.1016/S0140-6736(21)00869-2.

[38] Ferretti, Luca, Chris Wymant, Michelle Kendall, Lele Zhao, Anel Nurtay, Lucie AbelerDörner, Michael Parker, David Bonsall, and Christophe Fraser. "Quantifying SARSCoV-2 Transmission Suggests Epidemic Control with Digital Contact Tracing." Science 368, no. 6491 (May 8, 2020). https://doi.org/10.1126/science.abb6936.

[39] Ian M Mackay, 2020, The Swiss cheese model of pandemic defense, The New York Times, available from https://www.nytimes.com/2020/12/05/health/ coronavirus-swiss-cheese-infection-mackay.html (last accessed: 09 Aug 2021). 


\section{Supplementary Files}

This is a list of supplementary files associated with this preprint. Click to download.

- MasksonTrainstechsupplement.pdf 